\title{
Method for determining the maximum load of consumers in city power supply systems
}

\author{
A.G. Saidkhodjaev ${ }^{1 *}$, A.M. Najimova ${ }^{2}, A . K$. Bijanov $^{2}$ \\ ${ }^{1}$ Tashkent State Technical University named after Islam Karimov, 100095, Uzbekistan, Tashkent, University St. 2 A. \\ ${ }^{2}$ Karakalpak State University, 230100, Uzbekistan, Nukus, Ch. Abdirov St.1.
}

\begin{abstract}
In this article, we propose a new method for determining the maximum load of electric consumers in urban electric networks, which differs from existing methods in more accurate and reliable determination of the maximum loads. Based on the determination of the maximum loads of the objects of urban electrical networks, it is concluded that the proposed methods are determined by high accuracy and minor errors.
\end{abstract}

The rapid growth of social and technical development, the widespread computerization of all aspects of life in the Republic of Uzbekistan places at the forefront not only the reliability of electricity supply, but also the creation, re-equipment and reconstruction of the entire power supply system of cities. In recent years, with the growth of the people's welfare, the electrical load in urban networks has increased.

The main problem is the accurate and reliable determination of electrical loads of urban consumers at all levels of the power supply system, since electrical loads are the basis of all electrical engineering calculations. Existing methods for calculating electrical loads: demand factor, ordered diagrams, specific load, etc. they have a large calculation error, however the size largely determines the network configuration and the choice of its parameters.

The concept of design electrical load is taken as the maximum load in the time interval of half an hour. Professors A. Glazunov and B. Kudrin offer an hour maximum load, and the author justifies in urban networks the total electrical load on the outgoing feeders of transformer substations (TS), distribution points (RP) and so on. Have a daily schedule when overlapping more flat, have cross sections of wires and cables of large quantities and heating time $3 \mathrm{~T}_{0}$ more than 30 minutes and meet the hourly maximum load. [2]

The patented two new ways of determining the maximum (calculated) electrical loads based on the formula of the daily schedule curve for each type of consumer are proposed. To obtain characteristic (typical) daily schedules of electrical loads, numerous onsite experimental studies of electrical loads in residential and public buildings were carried out, with the right to select the objects to be examined and their number, as well as the required number of measurements to obtain initial data with a sufficient degree of accuracy. The measurement time is selected during periods of maximum load maximum, depending on the nature of electricity consumption, climatic factors and the type of consumers, are surveyed in winter and summer maximums. The required number of measurements to obtain reliable initial data for calculating the load of the same type of consumers, according to the Student's distribution, is within [1]:

$$
60<n<\infty
$$

Therefore, measurements were carried out continuously at the same point of the electrical network for 8 days and at 8 objects of the same type, i.e. 64 measurements at the same point of the consumer. The essence of the proposed integrated method for calculating electrical loads is as follows:

- first, on the basis of numerous experimental studies of urban power consumers and consumers, using the mathematical apparatus of probability theory, characteristic (typical) daily graphs of electrical loads (almost a complete set) of city power consumers and power consumers were constructed.

- further on the basis of these characteristic daily schedules are determined

the equations of the curve of the function of changing the characteristic (typical) daily power consumption with 30 and 60-minute maximum (interval) of each type of electrical consumer individually, using the methods of Lagrange, Newton, Stirling, Bessel, Chebyshev, with line functions, interval method and other.

Electrical load in any element of the urban network is a probabilistic value - a function of random variable electrical loads. Therefore, the calculation of urban electrical networks should be carried out by a probabilistic-statistical method. In this case, the probability values of electrical loads can be considered as numerical characteristics of statistical distributions. 
Thus, the formula is defined that describes the curve of the daily load curve, which closely matches the characteristic (typical) schedule. For each type of consumer, its own curve equation and its own calculation formula. This allows to determine the maximum (rated) electrical load at the low voltage levels of the electrical networks of the city's power supply system using a set of characteristic (typical) daily load patterns and the installed (nominal) power with probability $0.7-0.9$ to determine the 30 and 60 minute maximum electrical load, that is, the maximum (calculated) value of the electrical load and individual electrical consumers, using probabilisticstatistical calculation methods using the formula:

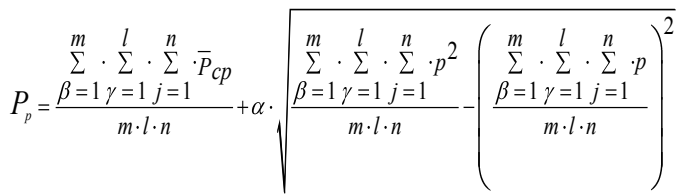

Where $\mathrm{P}_{\mathrm{p} \text {. }}$-average maximum of average maximum loads;

$\sigma$ - standard deviation;

$\alpha$ - normalized deviation

$\mathrm{n}$ - is the number of points of the measured value of the daily electrical load graph for the base time interval,

1 - is the number of days of load measurement at each point of the electrical network,

$\mathrm{m}$ - the number of similar objects of consumers.

For each type of consumer, its own curve equation, its own maximum (calculated) electrical load.

The total electrical load transformer substations (TS) distribution point (DP) we determine by summing up daily schedules of all consumers included in this network, for each half hour or hour (48 or 24 options), regardless of the number of electrical consumers included in this electric network, defined as the largest load of all summed periods, characteristic daily schedules with a maximum of electrical loads in a half-hour or hourly time interval (48 or 24 variants). [2]

The addition of active power must be carried out according to the probabilistic-statistical method for calculating electrical loads using the formula:

$$
\begin{aligned}
& \bar{P}_{\Sigma i}=C_{i 1} \bar{P}_{1}+C_{i 2} \bar{P}_{2}+C_{i 3} \bar{P}_{3}+\cdots+C_{i n} \bar{P}_{n}=\sum_{i=1}^{n} C_{i} \bar{P}_{i} \\
& \sigma_{\overline{P_{i}}}=\sqrt{C_{i 1}^{2} \sigma_{\bar{P} 1}^{2}+C_{i 2}^{2} \sigma_{\bar{P} 2}^{2}+\cdots+C_{i n}^{2} \sigma_{\bar{P} n}^{2}}
\end{aligned}
$$

where: $\mathrm{C}_{\mathrm{i}}$ - to the distribution coefficients of the schemes;

$\mathrm{Pi}$ - mathematical expectation (standard) of the load of the i-th consumer;

$\sigma_{p}$ - the standard deviation of the load of the i- th consumer.

The numerical characteristics of the full load will be equal to:

a) mathematical expectation

$$
\bar{S}=\sqrt{\bar{P}^{2}+\bar{Q}^{2}}
$$

b) standard deviation

$$
\sigma_{\bar{S}}=\sqrt{\cos ^{2} \bar{\varphi} \sigma_{\bar{P}}^{2}+\sin ^{2} \bar{\varphi} \sigma_{\bar{Q}}^{2}}
$$

When determining the total load of non-uniform consumers, the following expression is used for each calculated hour:

a) mathematical expectation

$$
\overline{S_{\Sigma}}=\sqrt{\left(\sum_{i=1}^{n} \overline{P_{i}}\right)^{2}+\left(\sum_{i=1}^{n} \overline{Q_{i}}\right)^{2}}
$$

b) standard deviation

$$
\sigma_{\overline{S_{\Sigma}}}=\sqrt{\cos _{\Sigma}^{2} \bar{\varphi} \sum_{i=1}^{n} \sigma_{\bar{P}_{i}}^{2}+\sin _{\Sigma}^{2} \bar{\varphi} \sum_{i=1}^{n} \sigma_{\bar{Q}_{i}}^{2}}
$$

\section{Conclusion}

1. New methods of calculation of electrical loads proposed by A. Saidkhodjaev. On the basis of numerous experiments, a complete set of typical daily schedules of electrical loads was developed. The method is developed and the formulas of the function of the curve of the daily schedule of electrical loads of the same type of consumers are obtained.

2. A method and technique for determining the total maximum (calculated) electrical load in the nodes of the power supply system of cities.

3. The program of calculation of schedules of daily loadings of consumers providing calculation of numerical characteristics and mathematical expectation and a standard deviation, daily loadings with half-hour intervals of time is created.

\section{References}

1. A.Saidkhodjaev, B.Teshabaev, Leu. Patent number IAP 04216 ( IAP 2006 0404), (2010).

2. A.Saidkhodjaev, Power supply of cities. Textbook. T.: "Fan va texnologiya", 328, (2015)

3. A.Saidkhodjaev, Fundamentals of energy saving. Textbook. - T.: "Lesson Press", 320, (2015).

4. A.Saidkhodjaev, Methods and tools of the energy audit. Textbook. - T.: "Noshirlik yog'dusi", 182, (2015). 5. A.Saidkhodjaev, Issues and prospects of urban power supply systems development. Monograph. - T.: "Fan", 256, (2012). 